\title{
Evaluation of faecal preservation and staining methods in the diagnosis of acute amoebiasis and giardiasis
}

\author{
N SHETTY, T PRABHU* Departments of Microbiology, St John's Medical College, Bangalore and the \\ *Victoria Hospital and Bangalore Medical College, Bangalore, India
}

SUMMARY The use of a faecal preservative and several staining methods, together with formalin ether concentration, were evaluated for the improved diagnosis of intestinal amoebiasis and giardiasis in 1285 patients with diarrhoea or dysentery and from asymptomatic controls. All samples were screened by three wet mount techniques. Thirty eight specimens of diarrhoeal or dysenteric stool were preserved in polyvinyl alcohol (PVA) and stained by trichrome and Spencer and Monroe short iron haematoxylin stain. Thirty nine preserved faecal samples submitted for routine screening were subjected to formalin ether concentration, wet mount examination, and permanent staining. Saline and buffered methylene blue (BMB) mounts were equally good for detection of trophozoite Entamoebae while Giardia trophozoites were detected only by the saline mount. The iodine mount was superior to the other mounts for protozoan cyst detection. The concentration procedure enhanced cyst recovery. Faecal preservation and subsequent staining was superior to wet mount examination for detection of the trophozoite stage and avoided the need for fresh specimens. Both the trichrome and the iron haematoxylin stains were comparable for the detection of cysts and trophozoites of the Entomoebae. Giardia lamblia trophozoites stained better with iron haematoxylin than with the trichrome.

Preservation and permanent staining is recommended as the most productive means for the accurate identification of the various protozoan parasites.

Diarrhoea due to protozoan parasites is an important cause of morbidity in developing countries.' Entamoeba histolytica and Giardia lamblia are two commonly implicated protozoans.

Diagnosis has depended largely on the demonstration of the trophozoite form in fresh faecal samples. Faecal examination for acute intestinal amoebiasis is complicated by the need to show active trophozoite motility, red cell inclusions, and characteristic nuclear morphology in fresh specimens. ${ }^{23}$ In giardiasis, demonstration of the motile trophic form in watery faeces remains the mainstay of diagnosis, though the presence of cysts in formed stool is adequate evidence of infection. Such criteria are considered to be mandatory before either protozoan is incriminated as the causative agent of gastrointestinal disease. ${ }^{23}$ This is of particular importance in the tropics where diarrhoeal or dysenteric episodes have multiple aetiology, and asymptomatic carriage of $E$ histolytica is known.' Though estimations of antibody titre have been widely

Accepted for publication 5 January 1988 used, both for amoebiasis and giardiasis, they are unreliable for diarrhoeal disease in endemic areas. ${ }^{45}$ Recent published work has shown that detection of faecal antigen is both sensitive and specific, ${ }^{67}$ but the reagents needed for these tests are expensive and not readily available in developing countries and hence are not widely used.

Several procedures have therefore been introduced, and evaluated. Stained wet mounts have been recommended because they delineate the nuclear characters of the Entamoebae in fresh faecal specimens. ${ }^{8}$ It may not always be possible to obtain fresh stool samples, however, nor is it possible for a microscopist to examine several fresh samples simultaneously. Stool preservatives were therefore introduced to eliminate the need for fresh specimens. Faecal examinations could be performed at leisure; furthermore, fragile trophic forms retained their integrity and distinctive morphological character. ${ }^{2}$ Subsequent staining and critical observation of nuclear characters with an oil immersion lens facilitated accurate diagnosis. ${ }^{3}$ This is especially important in gut protozoology where varia- 
tion in the trophozoite stages of the different protozoa can occur. ${ }^{3}$

This study was conducted in an area endemic for amoebiasis and giardiasis. Its purpose was to evaluate various staining methods both before and after faecal preservation in an attempt at accurate protozoan identification.

\section{Material and methods}

Clinical material was obtained from the two major hospitals of urban Bangalore: the Victoria Hospital and the St John's Medical College Hospital. A total of 1285 faecal specimens from outpatients were screened. These included samples from patients with acute intestinal disease as well as those submitted for routine screening. An iodine wet mount alone was used to screen 916 faecal samples out of the 1285. The remaining 369 specimens were categorised into two groups: (i) specimens of diarrhoeal or dysenteric stool $(n=38)$ from patients with acute gastrointestinal disease; and (ii) solid stool submitted for routine screening from patients with no history or acute illness $(n=331)$. Samples from both groups were subjected to a triple mount examination of fresh faecal material using $\mathbf{0 . 8 5 \%}$ sodium chloride, D'Antoni's iodine, and buffered methylene blue (BMB). ${ }^{9}$

As it is difficult to obtain or examine fresh specimens in a routine outpatient service the effectiveness of using polyvinyl alcohol (PVA) as a preservative was evaluated. Patients belonging to group (i)-that is, those presenting with watery, bloody, or mucoid diarrhoea-were given a phial containing $10-12 \mathrm{ml}$ of PVA preservative and fixative solution' for preservation of fresh stool specimens. Preservation was achieved soon after collection of the sample and before interfering substances such as drugs or barium salts were administered to the patient. After a time lag of three to four hours smears were made from the preserved stool and stained by two permanent stain techniques - the trichrome and the short iron haematoxylin stain of Spencer and Monroe.' An average of four to eight slides were made from each faecal specimen. Half the number of slides from each specimen were processed by the trichrome stain and the other half by the iron haematoxylin method. This was done to evaluate the reliability of the stains and the preservative to yield consistently good morphological detail in the differentiation of the various protozoa. All slides were screened using an oil immersion lens and graded as follows: $O=$ poor morphological detail; $+=$ fair amount of detail; $++=$ good morphological differentiation.

When comparing the two stains care was taken to see that other conditions such as age of specimen, time of fixation, and technique of staining were similarly standardised for both methods.

Thirty nine faecal samples from group ii were also preserved in PVA and subjected to formalin ether concentration $^{9}$ as well as permanent staining. Four smears were prepared from each specimen, two of which were stained by the trichrome method and the other two by the iron haematoxylin stain. All smears were viewed with an oil immersion lens and graded as above.

Results were analysed using the $\chi^{2}$ test.

\section{Results}

\section{WET MOUNT TECHNIQUES}

Table 1 indicates the recovery rate of protozoan parasites using only an iodine mount, compared with the triple mount technique of saline, iodine, and BMB. All trophozoite stages were missed using an iodine mount as the sole screening technique, but trophozoite stages of $E$ histolytica and $G$ lamblia were identified in $3 \%$ of the total number of samples examined using the triple mount technique.

Table 2 compares the efficacy of the saline, BMB, and iodine mounts for trophozoite and cyst detection.

Table 1 Comparison of D'Antoni's iodine mount and triple mount technique for recovering intestinal protozoa in fresh specimens

\begin{tabular}{|c|c|c|}
\hline Protozoan & $\begin{array}{l}\text { D'Antoni's } \\
\text { iodine mount } \\
\text { No }(\%)\end{array}$ & $\begin{array}{l}\text { Triple mount } \\
\text { technique } \dagger \\
\text { No }(\%)\end{array}$ \\
\hline \multicolumn{3}{|l|}{ E histolytica } \\
\hline $\begin{array}{l}\text { Trophozoite } \\
\text { Cyst } \\
\text { E coli }\end{array}$ & $\begin{array}{l}0 \\
66(7 \cdot 2)\end{array}$ & $\begin{array}{l}12(3 \cdot 3) \\
43(11 \cdot 7)\end{array}$ \\
\hline Trophozoite & 0 & $2(0 \cdot 5)$ \\
\hline \multicolumn{2}{|l|}{ G lamblia } & $12(3 \cdot 3)$ \\
\hline Trophozoite & 0 & $11(3 \cdot 0)$ \\
\hline Cyst & $11(1 \cdot 2)$ & $11 \quad(3.0)$ \\
\hline
\end{tabular}

No of slides screened: ${ }^{*} 916 ;+369$.

Table 2 Evaluation of saline, $B M B$, and iodine wet mounts for detecting $\mathrm{No}(\%)$ of trophozoites and cysts of protozoan parasites

\begin{tabular}{|c|c|c|c|c|}
\hline Protozoa & Saline & $B M B$ & Iodine & p value $\ddagger$ \\
\hline $\begin{array}{l}\text { E histolytica } \\
\text { Trophozoite* } \\
\text { Cyst } \dagger\end{array}$ & $\begin{array}{l}5(13 \cdot 2) \\
0\end{array}$ & $\begin{array}{l}12(31 \cdot 6) \\
31 \quad(9 \cdot 4)\end{array}$ & $\begin{array}{l}0 \\
29(8 \cdot 8)\end{array}$ & $\begin{array}{l}<0 \cdot 10 \\
\text { NS }\end{array}$ \\
\hline $\begin{array}{l}\text { E coli } \\
\text { Trophozoite } \\
\text { Cyst } \\
\text { G lamblia }\end{array}$ & $\begin{array}{ll}1 & (2 \cdot 6) \\
0 & \end{array}$ & $\begin{array}{l}2 \\
0\end{array}$ & $\begin{array}{l}0 \\
12(3 \cdot 6)\end{array}$ & $\begin{array}{l}\text { NS } \\
<0.005\end{array}$ \\
\hline $\begin{array}{l}\text { Trophozoite } \\
\text { Cyst }\end{array}$ & $\underset{0}{11}(28.9)$ & $\begin{array}{l}0 \\
0\end{array}$ & $\begin{array}{l}0 \\
6(1 \cdot 8)\end{array}$ & $\begin{array}{l}<0.005 \\
<0.02\end{array}$ \\
\hline
\end{tabular}

*Shows trophozoite detection in 38 diarrhoeal samples (group i). †Shows cyst detection in 331 samples of solid stool (group ii).

$\ddagger p$ values compare saline and BMB for detection of trophozoites and iodine with BMB for detection of cysts. 
There was no significant difference between the saline and BMB mounts in the detection of trophozoites of $E$ histolytica and $E$ coli. Though all trophic forms were rendered non-motile by BMB, the nuclear characters of the Entamoebae stained well with the dye. Identification of motile $G$ lamblia trophozoites was significantly better with saline, as they did not retain their morphological characteristics after staining with BMB. Iodine destroys all trophozoite forms, rendering them unrecognisable. Cyst detection by the iodine and BMB mounts is also shown in table 2. A comparative analysis showed that detection of $E$ histolytica cysts was comparable by either technique. The iodine mount was superior for the detection of $E$ coli and $G$ lamblia cysts as BMB did not stain these. Identification of cysts is not possible with saline as the nucleus is not visible.

FAECAL PRESER VATION, PERMANENT STAINING, AND FORMALIN ETHER CONCENTRATION

Detection of trophozoites by the saline mount using fresh faeces was compared with that using PVA preservation and subsequent staining with the trichrome and iron haematoxylin methods (table 3 ). Faecal preservation and permanent staining methods were far superior to the saline mount method for

Table 3 Comparison of faecal saline mounts and permanent stains for identification of $E$ histolytica and $G$ lamblia trophozoites from $36^{*}$ samples

\begin{tabular}{lcr}
\hline Screening method & $\begin{array}{l}\text { Ehistolytica } \\
\text { No (\%) }\end{array}$ & $\begin{array}{l}\text { G lamblia } \\
\text { No (\%) }\end{array}$ \\
\hline Saline & $5(13 \cdot 9)$ & $13(36 \cdot 1)$ \\
Iron-haematoxylin & $16(44 \cdot 4)$ & $12(33 \cdot 3)$ \\
Trichrome & $16(44 \cdot 4)$ & $3(8 \cdot 3)$ \\
p values $\dagger$ & $<0.005$ & $<0 \cdot 005$ \\
\hline
\end{tabular}

*Two of the 38 initially examined were later diagnosed as bacillary dysentery and ulcerative colitis respectively.

tp values compare saline and staining method for $E$ histolytica, and trichrome with iron haematoxylin for $G$ lamblia. detection of $E$ histolytica trophozoites. Detection of $G$ lamblia trophozoites was as effective as the saline mount and the iron haematoxylin stain on smears fixed in PVA. Faecal preservation, however, avoids the need for the immediate examination of fresh faecal material. The trichrome stain showed poor morphological detail of $G$ lamblia trophozoites compared with the iron haematoxylin stain, but PVA preservation did not affect the morphological detail of both trophozoite species, even after several days kept at room temperature.

Table 4 compares the efficiency of the two permanent staining techniques. The trichrome stain produced discernible morphological detail in $84 \%$ (grades + and ++ ) of the slides containing $E$ histolytica trophozoites; the iron haematoxylin stain yielded $69 \%$. Though there was no significant difference between the two stains; the trichrome stain produced a better contrast between nuclear and inclusion characteristics (staining red) against a blue-green background. Both stains stained $E$ coli trophozoites equally well. None of the slides containing trophozoites of $G$ lamblia was stained well by the trichrome method. In contrast, the iron haematoxylin stain successfully stained $G$ lamblia trophozoites in $61.5 \%$ of the slides. The smaller trophic forms such as $E$ hartmanni were more effectively stained by the iron haematoxylin method.

The larger and more mature cyst forms such as those of $E$ coli were not well preserved with PVA and hence stained poorly. The smaller and less mature cysts of the Entamoebae were better preserved and stained well by both methods. $G$ lamblia cysts were seen in about $65 \%$ of slides by either method, the trichrome stain yielding characteristic colour differentiation. Pus cell staining was comparable by both techniques.

Table 5 shows the efficacy of wet mount examination, formalin ether concentration, and permanent staining for the identification of the various protozoa. The permanent stains and the concentration procedure were equally efficient in their ability to

Table 4 No (\%) evaluation of trichrome and iron-haematoxylin stains for detection of protozoan parasites

\begin{tabular}{|c|c|c|c|c|c|c|c|c|c|}
\hline \multirow[b]{3}{*}{ Protozoa } & \multicolumn{4}{|c|}{ Trichrome } & \multicolumn{4}{|c|}{ Iron-haematoxylin } & \multirow[b]{3}{*}{ p Value } \\
\hline & \multirow{2}{*}{$\begin{array}{l}\text { No of } \\
\text { slides } \\
\text { screened }\end{array}$} & \multicolumn{3}{|l|}{ Grading } & \multirow{2}{*}{$\begin{array}{l}\text { No of } \\
\text { slides } \\
\text { screened }\end{array}$} & \multicolumn{3}{|l|}{ Grading } & \\
\hline & & 0 & + & ++ & & 0 & + & ++ & \\
\hline $\begin{array}{l}\text { E histolytica trophozoite } \\
\text { E coli trophozoite } \\
\text { G lamblia trophozoite } \\
\text { E hartmanni trophozoite } \\
\text { E histolytica cyst } \\
\text { E coli cyst } \\
\text { G lamblia cyst } \\
\text { E hartmanni cyst } \\
\text { Pus cells }\end{array}$ & $\begin{array}{r}75 \\
18 \\
26 \\
8 \\
18 \\
9 \\
15 \\
2 \\
22\end{array}$ & $\begin{array}{r}12(16 \cdot 0) \\
3(16 \cdot 6) \\
20(76 \cdot 9) \\
5(62 \cdot 5) \\
11(61 \cdot 1) \\
6(66 \cdot 6) \\
5(33 \cdot 3) \\
4(18 \cdot 2)\end{array}$ & $\begin{array}{cc}32 & (42 \cdot 6) \\
4 & (22 \cdot 2) \\
6 & (23 \cdot 1) \\
3 & (37 \cdot 5) \\
4 & (22 \cdot 2) \\
2 & (22 \cdot 2) \\
5 & (33 \cdot 3) \\
2 & (100) \\
4 & (18 \cdot 2)\end{array}$ & $\begin{array}{l}3(16 \cdot 6) \\
1(11 \cdot 1) \\
5(33.0) \\
4(18 \cdot 2)\end{array}$ & $\begin{array}{r}42 \\
18 \\
26 \\
8 \\
20 \\
7 \\
13 \\
4 \\
24\end{array}$ & $\begin{array}{r}13(31 \cdot 0) \\
2(11 \cdot 1) \\
10(38 \cdot 5) \\
1(12 \cdot 5) \\
8(40 \cdot 0) \\
3(42 \cdot 9) \\
4(30 \cdot 8) \\
3(12 \cdot 5)\end{array}$ & $\begin{array}{l}11(26 \cdot 2) \\
3(16 \cdot 6) \\
4(15 \cdot 4) \\
5(62 \cdot 5) \\
5(25 \cdot 0) \\
4(57 \cdot 1) \\
7(53 \cdot 8) \\
2(50 \cdot 0) \\
4(16 \cdot 6)\end{array}$ & $\begin{array}{r}18(42 \cdot 9) \\
13(72 \cdot 2) \\
12(46 \cdot 2) \\
2(25 \cdot 0) \\
7(35 \cdot 0) \\
2(15 \cdot 4) \\
2(50 \cdot 0) \\
17(70 \cdot 8)\end{array}$ & $\begin{array}{l}<0.10 \\
\text { NS } \\
<0.01 \\
<0.05 \\
<0.5 \\
<0.5 \\
\text { NS } \\
\text { NS } \\
<0.5\end{array}$ \\
\hline
\end{tabular}

0, poor detail; +, fair morphological detail; ++ , good morphological differentiation. 
Table 5 Comparison of triple mount technique, formalin ether concentration, and permanent staining methods for identifying intestinal protozoa

\begin{tabular}{|c|c|c|c|c|}
\hline \multirow[b]{2}{*}{ Protozoa } & \multirow[b]{2}{*}{$\begin{array}{l}\text { Triple wet mount } \\
\text { No }(\%)\end{array}$} & \multirow[b]{2}{*}{$\begin{array}{l}\text { Concentration } \\
\text { No }(\%)\end{array}$} & \multicolumn{2}{|l|}{ Permanent stains } \\
\hline & & & $\begin{array}{l}\text { Trichrome } \\
\text { No }(\%)\end{array}$ & $\begin{array}{l}\text { Iron } \\
\text { haematoxylin } \\
\text { No }(\%)^{*}\end{array}$ \\
\hline \multicolumn{5}{|l|}{ E histolytica } \\
\hline Trophozoite & 0 & 0 & $8(10.3)^{*} p<0.001$ & $8(10.3) p<0.001$ \\
\hline Cyst & $4(10 \cdot 3)$ & $10(25 \cdot 6)$ & $10(12 \cdot 8)$ & $10(12 \cdot 8)$ \\
\hline \multicolumn{5}{|l|}{ E coli } \\
\hline Trophozoite & 0 & 0 & $6(7 \cdot 3)$ & $6(7 \cdot 3)$ \\
\hline Cyst & $3(7 \cdot 7)$ & $4(10 \cdot 3)$ & $8(10 \cdot 3)$ & $8(10 \cdot 3)$ \\
\hline \multicolumn{5}{|l|}{ E hartmanni } \\
\hline Trophozoite & 0 & 0 & $2(2 \cdot 6)$ & $4(5 \cdot 1)$ \\
\hline \multicolumn{4}{|l|}{ G lamblia } & $2(2 \cdot 6)$ \\
\hline Trophozoite & 0 & & 0 & $2(2 \cdot 6)$ \\
\hline Cyst & 0 & $2(5 \cdot 1)$ & $8(10 \cdot 3)$ & $6(7 \cdot 3)$ \\
\hline
\end{tabular}

*No of slides stained: triple wet mount $n=39$, concentration $n=39$, Trichrome $n=78$, iron-haematoxylin $n=78$.

*P value compares concentration with trichrome and iron haematoxylin stains; $p$ values were insignificant for all other protozoa.

detail cyst morphology on material preserved in PVA. The larger and more mature cyst forms that did not stain well were detected by concentration while the smaller cysts were visualised in the stained smear. Only permanent staining detected trophozoites.

\section{Discussion}

Protozoan parasites such as $E$ histolytica have a unique characteristic; unless certain objective criteria are fulfilled, they cannot be identified in the aetiology of gastrointestinal disease. ${ }^{23}$ Nevertheless, the direct demonstration of the organism is still the first line of investigation in parasitic diarrhoea.

A wet mount in physiological saline has always been the mainstay of any initial laboratory examination, in the hope of detecting motile trophozoites with refractile inclusions. Unfortunately, this depends on the availability of fresh faecal material that has not been contaminated by substances such as drugs or barium salts. Non-motile amoebae in a saline mount are of poor diagnostic value as they are difficult to distinguish from macrophages or polymorphonuclear leucocytes. ${ }^{23}$

To help delineate the nuclear details of the trophic forms a drop of Nair's BMB has been advocated for wet mount staining. ${ }^{8}$ Though motility was considerably hampered, this dye was superior to the saline mount for the Entamoebae, due to its staining property. This was not so for the $G$ lamblia trophozoites, which became morphologically unidentifiable after BMB staining. Cystic stages of $E$ histolytica were also adequately stained by BMB, but $G$ lamblia cysts remained unstained. The only confusing aspect of the BMB staining is that polymorphs in the faeces can be mistaken for trophozoites of $E$ histolytica (Garcia LS, personal communication.) Hence a multi- ple wet mount using physiological saline, iodine, and BMB is recommended over a single mount to screen fresh faecal specimens for trophozoites and cysts. It is now well accepted, however, that the percentage of error in the exclusive examination of fresh specimens as wet mounts is too great for accurate diagnostic work. ${ }^{10}$

As the chances of obtaining fresh faecal material from the home, clinic, or hospital are remote the use of a faecal preservative needs to be evaluated. Although PVA precludes the use of a wet mount stain, its main advantage is that permanent smears and subsequent staining can be done on preserved material. PVA is also a good fixative and concentration procedures can also be done on material preserved in PVA. ${ }^{3}$ Preservation of the trophic stages of $E$ histolytica, E coli and $G$ lamblia were found to be of very high quality as were those of $G$ lamblia and $E$ histolytica cysts. In contrast, $E$ coli cysts were poorly or inconsistently preserved. In their early work with PVA, Brooke and Goldman showed the rapid and complete fixation of protruding pseudopods in dysenteric stool specimens. "They did not, however, unreservedly recommend PVA for the preservation of cysts.

Stained faecal films of fresh or PVA preserved material have been emphasised as being the single most productive means of stool examination for protozoa. ${ }^{10}$ In our study we found that the trichrome stain produced good contrast between nucleus, ingested red cells, and cytoplasm and hence showed excellent morphology for trophozoite stages of the Entamoebae (figure). This property, together with the fact that it keeps well, and the possibility of repeated reuse have been described by other workers. ${ }^{39}$ The iron haematoxylin stain, on the other hand, was better for smaller intestinal protozoa, particularly for the trophozoite stages of intestinal flagellates (figure): a 


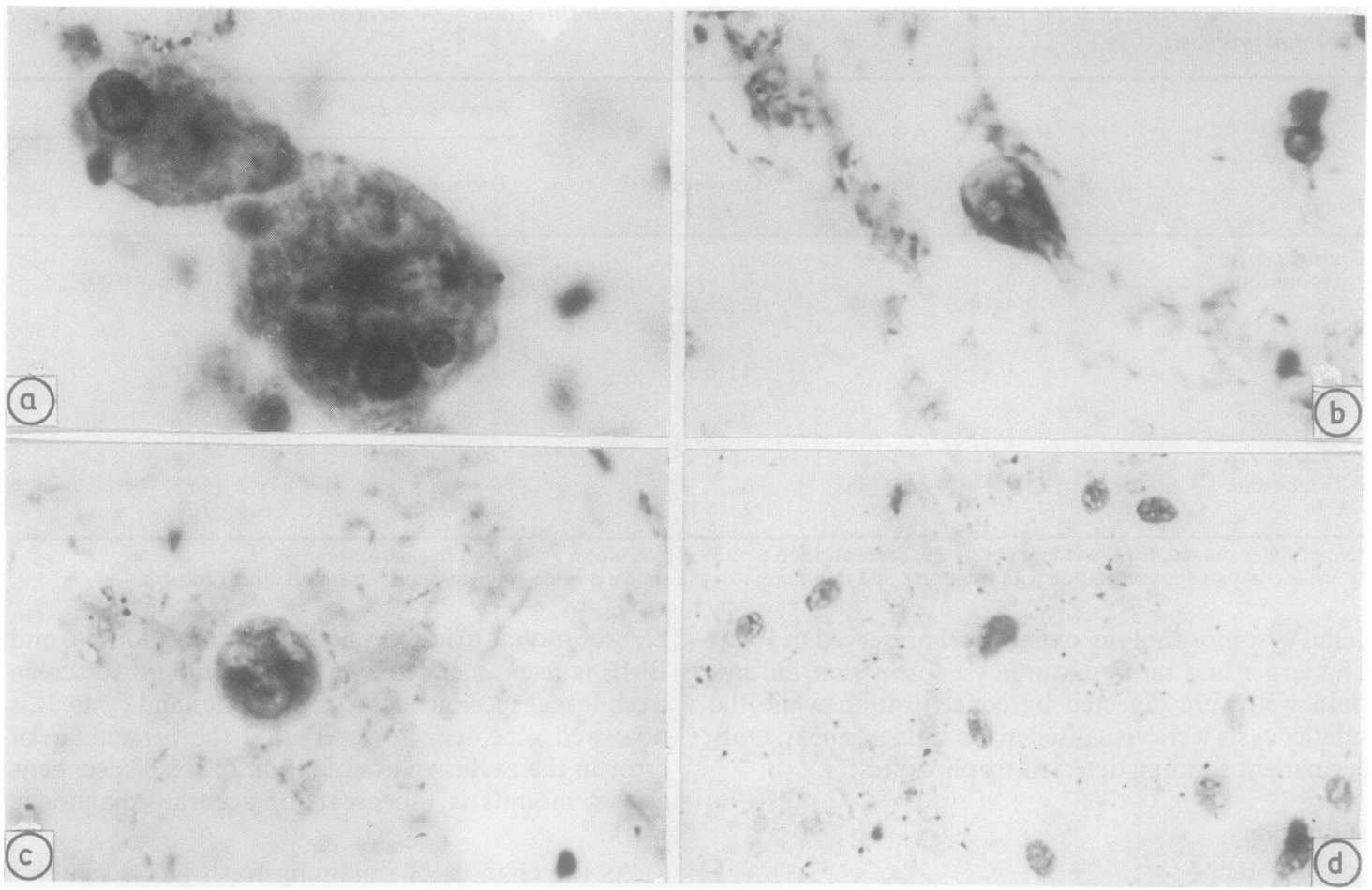

Figure (a) Trophozoite of E histolytica (trichrome stain); (b) trophozoite of G lamblia (iron haematoxylin stain); (c) cyst of E histolytica (trichrome stain); (d) cysts of G lamblia (trichrome stain). All stains were done on smears from faecal material preserved in PVA.

finding corroborated by Garcia and Voge. ${ }^{3}$ The disadvantages of the iron-haematoxylin stain are that it does not keep well and the procedure is more laborious. ${ }^{3}$ The cystic stages of $E$ histolytica and $G$ lamblia also stained well with the above techniques. None of the $E$ histolytica cysts showed the presence of chromatoid bars, characteristic of the early or precystic stage, but the nuclear morphological detail was adequate for accurate identification.

The formalin ether concentration is recommended as it is superior to a direct wet mount for the recovery of helminth eggs and protozoan cysts. This method does not concentrate the trophic forms and is therefore not useful in diagnosing dysenteries or diarrhoeas of protozoan origin, but the concentration procedure can be used to detect the cyst passer in $E$ histolytica and $G$ lamblia infestations. The smaller cysts, like those of $G$ lamblia, do not concentrate well on PVA preserved material; the larger more mature forms such as $E$ histolytica and $E$ coli cysts are easily detected by formalin ether concentration.

This study, as far as we know, is the first conducted in the tropics which evaluates faecal preservation and staining methods for improved diagnosis of protozoal diarrhoeas. Faecal preservation in kit form for use in an outpatient department is simple, effective, and inexpensive, and we recommend the routine preservation of all faecal samples. For a complete faecal examination, both the concentration and the staining methods are preferable, failing which, examination of the stained smear is the most effective means of enhancing recovery and identification of both the trophozoite and cyst forms of the various protozoan pathogens.

We are grateful to Dr JA Thomas, professor of pathology, St John's Medical College, for his attention to the photomicrography work and his close scrutiny of the manuscript. Our thanks are also due to $\mathrm{Dr}$ IM Thomas, professor of anatomy and cytogenetics, for permission to use the photomicrography equipment; to Dr HGV Rao for statistical analysis; to Dr Moire Jacob for her encouragement and enthusiasm; and to Ms Mary Queenie Lewis for secretarial help.

\section{References}

1 Davis A, Pawlowski ZS. Amoebiasis and its control. Bull WHO 1985;63:417-26. 
2 Healy GR. The laboratory diagnosis of amoebiasis. Bull N Y Acad Med 1971;47:478-93.

3 Garcia LS, Voge M. Diagnostic clinical parasitology I. Proper specimen collection and processing. Am J Med Technol 1980;46:459-67.

4 Healy GR. The use and limitations of the indirect haemagglutination test in the diagnosis of intestinal amoebiasis. Health Lab Sci 1968;5:174-9.

5 Goka AKJ, Rolston DDK, Mathan VI, Farthing MJG. Diagnosis of giardiasis by specific IgM antibody ELISA. Lancet 1986;ii:184-6.

6 Green EL, Miles MA, Warhurst DC. Immunodiagnostic detection of Giardia antigen in faeces by a rapid visual enzyme linked immunosorbent assay. Lancet 1985;ii:691-3.

7 Grundy MS, Voller A, Warhurst D. An enzyme-linked immunosorbent assay for the detection of Entamoeba histolytica antigens in faecal material. Trans $R$ Soc Trop Med Hyg 1987;81:627-32.
8 Nair CP. Rapid staining of intestinal amoebae on wet mounts. Nature 1953;172:1051.

9 Garcia LS. Laboratory diagnosis of parasitic infections. In: Finegold SM, Martin WJ, eds. Diagnostic microbiology. St Louis: The CV Mosby Co, 1982:457-75.

10 Garcia LS, Brewer TC, Bruckner DA. A comparison of the formalin ether concentration and trichrome stained smear methods for the recovery and identification of intestinal protozoa. Am J Med Technol 1979;45:932-5.

11 Brooke MM, Goldman M. Polyvinyl alcohol fixative as a preservative and adhesive for protozoa in dysenteric stools and other liquid material. J Lab Clin Med 1949;34:1554-60.

Requests for reprints to: Dr N Shetty, Department of Microbiology, St John's Medical School, Bangalore 560 034, India. 